\title{
Generalizations of Troisi's inequality in weighted $p$-Sobolev spaces with singularities
}

Hua Chen ${ }^{1 *}$, Yong Luo' and Jing Wang'

\section{"Correspondence:}

chenhua@whu.edu.cn

${ }^{1}$ School of Mathematics and

Statistics and Computational

Science Hubei Key Laboratory,

Wuhan University, Wuhan, China

\begin{abstract}
We extend classical Troisi's inequality to the weighted $p$-Sobolev spaces on stretched cone, edge, and corner respectively. The results here can be used to investigate anisotropic elliptic equations involving cone degeneracy, edge degeneracy, and corner degeneracy, which will be studied in our forthcoming papers.
\end{abstract}

MSC: $35 \mathrm{~J} 70 ; 35 \mathrm{~J} 75 ; 46 \mathrm{E} 35$

Keywords: Troisi's inequality; Weighted p-Sobolev space; Cone singularity; Edge singularity; Corner singularity

\section{Introduction and main results}

In 1969, M. Troisi (cf. [1]) found an important inequality. Its classical form can be described as: given $1 \leq p_{i}<\infty, i=1, \ldots, n$, for a smooth function $u$ compactly supported in $\mathbb{R}^{n}$, the following inequality holds:

$$
\|u\|_{s} \leq C \prod_{i=1}^{n}\left\|\partial_{x_{i}} u\right\|_{p_{i}}^{\frac{1}{n}}, \quad \sum_{i=1}^{n} \frac{1}{p_{i}}>1, s=\frac{n}{\sum_{i=1}^{n} \frac{1}{p_{i}}-1},
$$

where $\|u\|_{q}=\left(\int_{\mathbb{R}^{n}}|u|^{q} \mathrm{~d} x\right)^{\frac{1}{q}}$ with $1 \leq q<\infty$ and $C$ is independent of $u$. It is the well-known Troisi's inequality that can be used to study the existence of multiple nonnegative solutions to the anisotropic critical problem (cf. [2])

$$
-\sum_{i=1}^{n} \frac{\partial}{\partial x_{i}}\left(\left|\frac{\partial u}{\partial x_{i}}\right|^{p_{i}-2} \frac{\partial u}{\partial x_{i}}\right)=|u|^{s-2} u, \quad \text { in } \mathbb{R}^{n},
$$

where $1<p_{i}<\infty$ for $i=1,2, \ldots, n, \sum_{i=1}^{n} \frac{1}{p_{i}}>1, s=n /\left(\sum_{i=1}^{n} \frac{1}{p_{i}}-1\right)$ is anisotropic critical exponent and $\max _{1 \leq i \leq n}\left\{p_{i}\right\}<s$. Applications of (1.1) also can be found in [3] to study the existence of fundamental solutions to anisotropic elliptic equations. Another generalization of (1.1) in [4] is used to prove regularity of the weak solution to the Navier-Stokes equations based on one component of velocity. By arithmetic and geometric mean inequality, (1.1) becomes an anisotropic Sobolev inequality presented as

$$
\|u\|_{s} \leq \frac{C}{n} \sum_{i=1}^{n}\left\|\partial_{x_{i}} u\right\|_{p_{i}}, \quad \sum_{i=1}^{n} \frac{1}{p_{i}}>1, s=\frac{n}{\sum_{i=1}^{n} \frac{1}{p_{i}}-1} .
$$

(c) The Author(s) 2019. This article is distributed under the terms of the Creative Commons Attribution 4.0 International License (http://creativecommons.org/licenses/by/4.0/), which permits unrestricted use, distribution, and reproduction in any medium, provided you give appropriate credit to the original author(s) and the source, provide a link to the Creative Commons license, and indicate if changes were made. 
In particular, if $p_{i}=p$ in (1.3) for $i=1, \ldots, n$, then (1.1) finally reduces to the classical Gagliardo-Nirenberg-Sobolev inequality

$$
\|u\|_{s} \leq \frac{C}{n} \sum_{i=1}^{n}\left\|\partial_{x_{i}} u\right\|_{p}, \quad 1 \leq p<n, s=\frac{n p}{n-p} .
$$

Here the methods to prove (1.3) and (1.4) are similar as that in Adams and Vancouver [5] by using mixed norms and permutation inequalities and that in Kružkov [6, p. 282] to establish a new proof based on fundamental theorem of calculus.

Motivations of this paper mainly come from the attention to studying the anisotropic elliptic equations as (1.2) with conical singularity, edge singularity, and corner singularity respectively. For instance, (1.2) including conical singularity corresponds to

$$
-\sum_{i=1}^{n} D_{c, i}\left(\left|D_{c, i} u\right|^{p_{i}-2} D_{c, i} u\right)=|u|^{s-2} u \text { in } \mathbb{R}_{+}^{n+1},
$$

where $D_{c, 0}=t \partial_{t}, D_{c, i}=\partial_{x_{i}}, 1<p_{i}<\infty$ for $i=0,1,2, \ldots, n$, and anisotropic critical exponent $s:=(n+1) /\left(\sum_{i=0}^{n} \frac{1}{p_{i}}-1\right)$. As indicated above, the anisotropic elliptic equations with singularities of edge type or corner type parallel to (1.2) can be formulated as well.

Considering the pivotal role of Troisi's inequality in studying such kinds of singular anisotropic elliptic equations like (1.5) (e.g., see the results in our upcoming papers), we need, in the first place, to deduce it being of different forms in different weighted $p$-Sobolev spaces. To be specific, we will generalize (1.1) to some singular weighted $p$-Sobolev spaces (see Sect. 2 below) in which the usual gradient operator $\nabla=\left(\partial_{x_{1}}, \partial_{x_{2}}, \ldots, \partial_{x_{n}}\right)$ becomes the cone type, edge type, and corner type gradient operators such as $D_{c}=$ $\left(t \partial_{t}, \partial_{x_{1}}, \ldots, \partial_{x_{n}}\right)$ in $\mathbb{R}_{+}^{n+1}, \mathrm{D}_{e}=\left(t \partial_{t}, \partial_{x_{1}}, \partial_{x_{2}}, \ldots, \partial_{x_{n}}, t \partial_{y_{n+1}}, \ldots, t \partial_{y_{n+q}}\right)$ in $\mathbb{R}_{+} \times \mathbb{R}^{n} \times \mathbb{R}^{q}$, and $\mathrm{D}_{\text {cor }}=\left(r \partial_{r}, \partial_{x_{1}}, \partial_{x_{2}}, \ldots, \partial_{x_{n}}, r t \partial_{t}\right)$ in $\mathbb{R}_{+} \times \mathbb{R}^{n} \times \mathbb{R}_{+}$respectively. Now we present our main conclusions of this paper as follows.

Theorem 1.1 Let $\gamma \in \mathbb{R}, 1 \leq p_{i}<\infty$ for $0 \leq i \leq n$, and $\sum_{i=0}^{n} \frac{1}{p_{i}}>1$. Set $\frac{1}{s}=\frac{1}{n+1}\left(\sum_{i=0}^{n} \frac{1}{p_{i}}-1\right)$. Then we have the following cone type Troisi's inequality for all $u(t, x) \in C_{0}^{\infty}\left(\mathbb{R}_{+}^{n+1}\right)$ :

$$
\|u\|_{L_{s}^{\gamma}} \leq\left(c_{01}\|u\|_{L_{p_{0}}^{\gamma_{*}^{*}}}^{\frac{1}{n+1}}+c_{02}\left\|t \partial_{t} u\right\|_{L_{p_{0}}^{\gamma^{*}}}^{\frac{1}{n+1}}\right) \prod_{i=1}^{n} c_{i}\left\|\partial_{x_{i}} u\right\|_{L_{p_{i}}^{\gamma_{*}^{*}}}^{\frac{1}{n+1}}
$$

where $\|u\|_{L_{p}^{\gamma}}=\left(\int_{\mathbb{R}^{n}} \int_{\mathbb{R}_{+}} t^{n+1}\left|t^{-\gamma} u(t, x)\right|^{p} \frac{\mathrm{d} t}{t} \mathrm{~d} x\right)^{\frac{1}{p}}, \gamma_{j}^{*}=-\left(\frac{n+1}{s}-\gamma-\frac{n+1}{p_{j}}\right)$ for $0 \leq j \leq n, c_{01}=$ $\frac{1}{2}\left[\left(1+\frac{s\left(p_{0}-1\right)}{p_{0}}\right)\left|\frac{n+1}{s}-\gamma\right|\right]^{\frac{1}{n+1}}, c_{02}=\frac{1}{2}\left(1+\frac{s\left(p_{0}-1\right)}{p_{0}}\right)^{\frac{1}{n+1}}$, and $c_{i}=\left(1+\frac{s\left(p_{i}-1\right)}{p_{i}}\right)^{\frac{1}{n+1}}$ for $1 \leq i \leq n$.

Moreover, as a special case, we obtain the following cone type Sobolev inequality which was first proved by [7, Theorem 2.1] in studying Dirichlet problem for nonlinear elliptic boundary value problem on a manifold with conical singularities. 
Corollary 1.2 In addition to the conditions included in Theorem 1.1, if $p_{i}=p \geq 1$ for $0 \leq$ $i \leq n$, then we have the following cone type Sobolev inequality:

$$
\|u\|_{L_{s}^{\gamma}} \leq \hat{c}_{0}\|u\|_{L_{p}^{\gamma+1}}+\hat{c}_{1}\left(\left\|\left(t \partial_{t}\right) u\right\|_{L_{p}^{\gamma+1}}+\sum_{i=1}^{n}\left\|\partial_{x_{i}} u\right\|_{L_{p}^{\gamma+1}}\right)
$$

where $\hat{c}_{0}=\frac{n|n+1-p(\gamma+1)|}{2(n+1)(n+1-p)}, \hat{c}_{1}=\frac{n p}{2(n+1)(n+1-p)}, \frac{1}{s}=\frac{1}{p}-\frac{1}{n+1}$, and $p<n+1$.

Secondly, we consider the following edge type Torisi's inequality.

Theorem 1.3 Given $1 \leq p_{i}<\infty$ for $0 \leq i \leq n+q$, and $\sum_{i=0}^{n+q} \frac{1}{p_{i}}>1$. Let $\frac{1}{s}=\frac{1}{n+q+1}\left(\sum_{i=0}^{n+q} \frac{1}{p_{i}}-\right.$ 1). Then we have the following edge type Troisi's inequality for all $u(t, x, y) \in C_{0}^{\infty}\left(\mathbb{R}_{+} \times \mathbb{R}^{n} \times\right.$ $\left.\mathbb{R}^{q}\right)$ and $\gamma \in \mathbb{R}:$

$$
\begin{aligned}
\|u\|_{\mathcal{L}_{s}^{\gamma}} \leq & \left(c_{01}\|u\|_{\mathcal{L}_{p_{0}}^{\gamma_{0}^{*}}}^{\frac{1}{n+q+1}}+c_{02}\left\|t \partial_{t} u\right\|_{\mathcal{L}_{p_{0}}^{\gamma_{0}^{*}}}^{\frac{1}{n+q+1}}\right) \prod_{i=1}^{n} c_{i}\left\|\partial_{x_{i}} u\right\|_{\mathcal{L}_{p_{i}}^{\gamma_{i}^{*}}}^{\frac{1}{n+q+1}} \\
& \times \prod_{i=n+1}^{n+q} c_{i}\left\|\left(t \partial_{y_{i}}\right) u\right\|_{\mathcal{L}_{p_{i}}^{\gamma_{i}^{*}}}^{\frac{1}{n+q+1}}
\end{aligned}
$$

where $\|u\|_{\mathcal{L}_{p}^{\gamma}}=\left(\int_{\mathbb{R}_{+}^{N}} t^{n+q+1}\left|t^{-\gamma} u(t, x, y)\right|^{p} \frac{\mathrm{d} t}{t} \mathrm{~d} x \frac{\mathrm{d} y}{t}\right)^{\frac{1}{p}}, \gamma_{j}^{*}=-\left(\frac{n+q+1}{s}-\gamma-\frac{n+q+1}{p_{j}}\right)$ for $0 \leq j \leq n+$ $q, c_{01}=\frac{1}{2}\left[\left(1+\frac{s\left(p_{0}-1\right)}{p_{0}}\right)\left|\frac{n+q+1}{s}-\gamma\right|\right]^{\frac{1}{n+q+1}}, c_{02}=\frac{1}{2}\left(1+\frac{s\left(p_{0}-1\right)}{p_{0}}\right)^{\frac{1}{n+q+1}}$, and $c_{i}=\left(1+\frac{s\left(p_{i}-1\right)}{p_{i}}\right)^{\frac{1}{n+q+1}}$ for $1 \leq i \leq n+q$.

In particular, the following edge type Sobolev inequality can be regarded as a special case of the edge type Troisi's inequality above. This kind of edge type Sobolev inequality was first given by [8, Proposition 3.2] in studying Dirichlet problem for semilinear edgedegenerate elliptic equations.

Corollary 1.4 Under the assumptions in Theorem 1.3, if $p_{i}=p \geq 1$ for $0 \leq i \leq n+q$, then we have the following edge type Sobolev inequality:

$$
\|u\|_{\mathcal{L}_{s}^{\gamma}} \leq \hat{c}_{0}\|u\|_{\mathcal{L}_{p}^{\gamma+1}}+\hat{c}_{1}\left(\left\|\left(t \partial_{t}\right) u\right\|_{\mathcal{L}_{p}^{\gamma+1}}+\sum_{i=1}^{n}\left\|\partial_{x_{i}} u\right\|_{\mathcal{L}_{p}^{\gamma+1}}+\sum_{i=n+1}^{n+q}\left\|\left(t \partial_{y_{i}}\right) u\right\|_{\mathcal{L}_{p}^{\gamma+1}}\right)
$$

where $\hat{c}_{0}=\frac{(n+q)|n+q+1-p(\gamma+1)|}{2(n+q+1)(n+q+1-p)}$ and $\hat{c}_{1}=\frac{(n+q) p}{2(n+q+1)(n+q+1-p)}, \frac{1}{s}=\frac{1}{p}-\frac{1}{n+q+1}$ and $p<n+q+1$.

Finally, we give the corner type Troisi's inequality.

Theorem 1.5 If $1 \leq p_{i}<\infty$ for $0 \leq i \leq n+1, \sum_{i=0}^{n+1} \frac{1}{p_{i}}>1, \frac{1}{s}=\frac{1}{n+2}\left(\sum_{i=0}^{n+1} \frac{1}{p_{i}}-1\right)$ and $\bar{\gamma}, \gamma \in$ $\mathbb{R}$, then the following corner type Troisi's inequality holds for all $u(r, x, t) \in C_{0}^{\infty}\left(\mathbb{R}_{+} \times \mathbb{R}^{n} \times\right.$ $\left.\mathbb{R}_{+}\right)$:

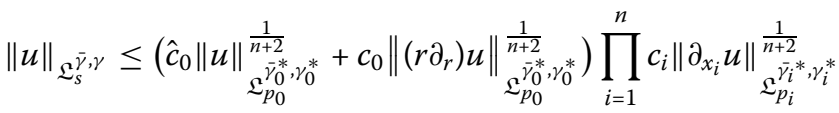

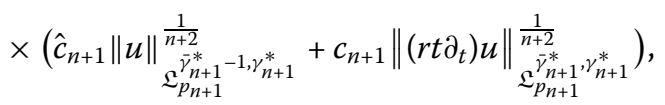


where $\|u\|_{\mathfrak{L}_{p}^{\gamma_{1}, \gamma_{2}}}=\left(\int_{\mathbb{R}_{+} \times \mathbb{R}^{n} \times \mathbb{R}_{+}}\left|r^{\frac{n+2}{p}-\gamma_{1}} t^{\frac{n+2}{p}-\gamma_{2}} u(r, x, t)\right|^{p} \frac{\mathrm{d} r}{r} \mathrm{~d} x \frac{\mathrm{d} t}{r t}\right)^{\frac{1}{p}}, \bar{\gamma}_{i}^{*}=-\left(\frac{n+2}{s}-\bar{\gamma}-\frac{n+2}{p_{i}}\right), \gamma_{i}^{*}=$ $-\left(\frac{n+2}{s}-\gamma-\frac{n+2}{p_{i}}\right), c_{0}=\frac{1}{2}\left(1+\frac{s\left(p_{0}-1\right)}{p_{0}}\right)^{\frac{1}{n+2}}, c_{i}=\left(1+\frac{s\left(p_{i}-1\right)}{p_{i}}\right)^{\frac{1}{n+2}}$ for $1 \leq i \leq n+1, \hat{c}_{0}=\frac{1}{2}[(1+$ $\left.\left.\frac{s\left(p_{0}-1\right)}{p_{0}}\right)\left|\frac{n+2}{s}-\bar{\gamma}\right|\right]^{\frac{1}{n+2}}, \hat{c}_{n+1}=\left[\left(1+\frac{s\left(p_{n+1}-1\right)}{p_{n+1}}\right)\left|\frac{n+2}{s}-\gamma\right|\right]^{\frac{1}{n+2}}$.

Likewise, it follows from Theorem 1.5 that we can derive the corner type Sobolev inequality as follows, which was first obtained by [9, Proposition 3.1] in studying the existence of multiple solutions for semi-linear corner degenerate elliptic equations.

Corollary 1.6 Based on Theorem 1.5, further if we choose $p_{i}=p \geq 1$ for $0 \leq i \leq n+1$, then we have the following corner type Sobolev inequality:

$$
\begin{aligned}
\|u\|_{\mathfrak{L}_{s}^{\bar{\gamma}, \gamma}} \leq & \tilde{\mu}_{0}\|u\|_{\mathcal{L}_{p}^{\bar{\gamma}+1, \gamma+1}}+\mu_{0}\left\|\left(r \partial_{r}\right) u\right\|_{\mathfrak{L}_{p}^{\bar{\gamma}+1, \gamma+1}}+\sum_{i=1}^{n} \mu_{i}\left\|\partial_{x_{i}} u\right\|_{\mathcal{L}_{p}^{\bar{\gamma}+1, \gamma+1}} \\
& +\hat{\mu}_{n+1}\|u\|_{\mathfrak{L}_{p}^{\bar{\gamma}, \gamma+1}}+\mu_{n+1}\left\|\left(r t \partial_{t}\right) u\right\|_{\mathfrak{L}_{p}^{\bar{\gamma}+1, \gamma+1}}
\end{aligned}
$$

where $\tilde{\mu}_{0}=\frac{(n+1)|n+2-p(\bar{\gamma}+1)|}{2(n+2)(n+2-p)}, \mu_{i}=\frac{p(n+1)}{2(n+2)(n+2-p)}$ for $0 \leq i \leq n+1, \hat{\mu}_{n+1}=\frac{(n+1)|n+2-p(\gamma+1)|}{2(n+2)(n+2-p)}, \frac{1}{s}=$ $\frac{1}{p}-\frac{1}{n+2}$ and $p<n+2$.

The outline of this paper is as follows. In Sect. 2, we introduce cone type, edge type, and corner type weighted $p$-Sobolev spaces respectively. Then, in Sect. 3, we give the proof of Theorem 1.1. Finally, the proofs of Theorem 1.3 and Theorem 1.5 will be provided in Sect. 4.

\section{Definitions of singular weighted $p$-Sobolev spaces}

Let $X$ be a closed compact $C^{\infty}$ manifold and $X^{\Delta}=\left(\overline{\mathbb{R}}_{+} \times X\right) /(\{0\} \times X)$ be a local model considered as a cone with the base $X$. In particular, let $X \subset S^{n}$ be a bounded open set in the unit sphere of $\mathbb{R}^{n+1}$, and the straight cone $X^{\Delta}$ is defined as

$$
X^{\Delta}=\left\{\tilde{x} \in \mathbb{R}^{n+1}: \tilde{x}=0 \text { or } \frac{\tilde{x}}{|\tilde{x}|} \in X\right\} .
$$

Thus, $X^{\wedge}=\mathbb{R}_{+} \times X$ is called as corresponding open stretched cone with the base $X$. In local coordinates, $\mathbb{R}_{+} \times \mathbb{R}^{n}$ can be interpreted as an open stretched cone. The typical differential operators, defined on a manifold with conical singularities, are called Fuchs type, i.e.,

$$
A=t^{-m} \sum_{k=0}^{m} a_{k}(t)\left(-t \partial_{t}\right)^{k}=t^{-m} A_{\mathbb{X}^{\Delta}},
$$

where $(t, x) \in \mathbb{R}_{+} \times \mathbb{R}^{n}, a_{k}(t) \in C^{\infty}\left(\overline{\mathbb{R}}_{+}, \operatorname{Diff}^{m-k}\left(\mathbb{R}^{n}\right)\right)$, Diff ${ }^{j}\left(\mathbb{R}^{n}\right)$ refers to the set of differential operators of order $j$ on $\mathbb{R}^{n}$, and $A_{\mathbb{X}^{\Delta}}$ are called degenerated cone operators.

Let $g$ be Riemannian metrics on $\mathbb{R}_{+} \times \mathbb{R}^{n}$, then

$$
g:=\mathrm{d} t^{2}+t^{2} \mathrm{~d} x^{2}=t^{2}\left[\left(\frac{\mathrm{d} t}{t}\right)^{2}+\mathrm{d} x^{2}\right]
$$

Hence the cone type gradient operator here is defined as $D_{c}:=\left(t \partial_{t}, \partial_{x_{1}}, \ldots, \partial_{x_{n}}\right)$. Now we introduce the following cone type weighted $L_{p}$-spaces. 
Definition 2.1 For $(t, x) \in \mathbb{R}_{+}^{n+1}\left(:=\mathbb{R}_{+} \times \mathbb{R}^{n}\right), 1 \leq p<+\infty$, and $u(t, x)$ in distribution space $\mathcal{D}^{\prime}\left(\mathbb{R}_{+}^{n+1}\right)$, then we consider that $u(t, x) \in L_{p}\left(\mathbb{R}_{+}^{n+1}, \frac{\mathrm{d} t}{t} \mathrm{~d} x\right)$ if

$$
\|u\|_{L_{p}\left(\mathbb{R}_{+}^{n+1}\right)}=\left(\int_{\mathbb{R}^{n}} \int_{\mathbb{R}_{+}} t^{n+1}|u(t, x)|^{p} \frac{\mathrm{d} t}{t} \mathrm{~d} x\right)^{\frac{1}{p}}<+\infty .
$$

Furthermore, the weighted cone type $L_{p}$-spaces with weight data $\gamma \in \mathbb{R}$ are denoted by $L_{p}^{\gamma}\left(\mathbb{R}_{+}^{n+1}, \frac{\mathrm{d} t}{t} \mathrm{~d} x\right)$. Namely, if $u(t, x) \in L_{p}^{\gamma}\left(\mathbb{R}_{+}^{n+1}, \frac{\mathrm{d} t}{t} \mathrm{~d} x\right)$, then $t^{-\gamma} u(t, x) \in L_{p}\left(\mathbb{R}_{+}^{n+1}, \frac{\mathrm{d} t}{t} \mathrm{~d} x\right)$, and

$$
\|u\|_{L_{p}^{\gamma}\left(\mathbb{R}_{+}^{n+1}\right)}=\left(\int_{\mathbb{R}^{n}} \int_{\mathbb{R}_{+}} t^{n+1}\left|t^{-\gamma} u(t, x)\right|^{p} \frac{\mathrm{d} t}{t} \mathrm{~d} x\right)^{\frac{1}{p}}<+\infty .
$$

Now we give the definition of singular weighted $p$-Sobolev spaces on stretched cone $\mathbb{R}_{+} \times \mathbb{R}^{n}$ as follows (cf. [7]).

Definition 2.2 For $\gamma \in \mathbb{R}, m \in \mathbb{N}$, and $1 \leq p<+\infty$, the singular weighted $p$-Sobolev spaces are defined as

$$
H_{p}^{m, \gamma}\left(\mathbb{R}_{+}^{n+1}\right):=\left\{u \in \mathcal{D}^{\prime}\left(\mathbb{R}_{+}^{n+1}\right):\left(t \partial_{t}\right)^{\alpha} \partial_{x}^{\beta} u(t, x) \in L_{p}^{\gamma}\left(\mathbb{R}_{+}^{n+1}, \frac{\mathrm{d} t}{t} \mathrm{~d} x\right)\right\}
$$

for arbitrary $\alpha \in \mathbb{N}, \beta \in \mathbb{N}^{n}$, and $|\alpha|+|\beta| \leq m$.

Moreover, the spaces $H_{p}^{m, \gamma}\left(\mathbb{R}_{+}^{n+1}\right)$ will be Banach spaces endowed with the norm

$$
\|u\|_{H_{p}^{m, \gamma}\left(\mathbb{R}_{+}^{n+1}\right)}=\sum_{|\alpha|+|\beta| \leq m}\left(\iint_{\mathbb{R}_{+}^{n+1}} t^{n+1}\left|t^{-\gamma}\left(t \partial_{t}\right)^{\alpha} \partial_{x}^{\beta} u(t, x)\right|^{p} \frac{\mathrm{d} t}{t} \mathrm{~d} x\right)^{\frac{1}{p}} .
$$

Next, we introduce the following edge type $p$-Sobolev spaces. First, we assume $X^{\Delta}$ is a straight cone, then for a bounded domain $Y$ in $\mathbb{R}^{q}, W:=X^{\Delta} \times Y$ is a corresponding wedge in $\mathbb{R}^{1+n+q}$. Thus the stretched wedge $\mathbb{W}$ to $W$ is $\overline{\mathbb{R}}_{+} \times X \times Y$, which is a manifold with smooth boundary $\{0\} \times X \times Y$. In local coordinates, the open stretched wedge will be $\mathbb{R}_{+} \times \mathbb{R}^{n} \times \mathbb{R}^{q}$.

The typical degenerate differential operator on the open stretched wedge $\mathbb{R}_{+} \times \mathbb{R}^{n} \times \mathbb{R}^{q}$ has the form of

$$
t^{-v} \sum_{j+|\alpha| \leq v} a_{j \alpha}(t, y)\left(t \partial_{t}\right)^{j}\left(t \partial_{y}\right)^{\alpha}=t^{-v} A_{\mathbb{W}}
$$

where $A_{\mathbb{W}}$ is a degenerate edge operator, $a_{j \alpha} \in C^{\infty}\left(\mathbb{R}_{+} \times \mathbb{R}^{q}\right.$, Diff $\left.{ }^{\nu-(j+|\alpha|)}\left(\mathbb{R}^{n}\right)\right)$ for all $j, \alpha$, and $\operatorname{Diff}^{i}\left(\mathbb{R}^{n}\right)$ denotes the set of differential operators of order $i$ on $\mathbb{R}^{n}$.

Furthermore, let $g$ be Riemannian metrics on $\mathbb{R}_{+} \times \mathbb{R}^{n} \times \mathbb{R}^{q}$. Then

$$
g:=\mathrm{d} t^{2}+t^{2} \mathrm{~d} x^{2}+\mathrm{d} y^{2}=t^{2}\left[\left(\frac{\mathrm{d} t}{t}\right)^{2}+\mathrm{d} x^{2}+\left(\frac{\mathrm{d} y}{t}\right)^{2}\right] .
$$

Thus, the edge type gradient operator is defined as $\mathrm{D}_{e}=\left(t \partial_{t}, \partial_{x_{1}}, \partial_{x_{2}}, \ldots, \partial_{x_{n}}, t \partial_{y_{n+1}}, \ldots\right.$, $\left.t \partial_{y_{n+q}}\right)$. At present, we give the following definition of edge type weighted $\mathcal{L}_{p}$-spaces. 
Definition 2.3 Assume $N=1+n+q,(t, x, y) \in \mathbb{R}_{+}^{N}\left(:=\mathbb{R}_{+} \times \mathbb{R}^{n} \times \mathbb{R}^{q}\right)$, and $u(t, x, y) \in$ $\mathcal{D}^{\prime}\left(\mathbb{R}_{+}^{N}\right)$. We consider that $u(t, x, y) \in \mathcal{L}_{p}\left(\mathbb{R}_{+}^{N}, \frac{\mathrm{d} t}{t} \mathrm{~d} x \frac{\mathrm{d} y}{t}\right)$ if

$$
\|u\|_{\mathcal{L}_{p}}=\left(\int_{\mathbb{R}_{+}^{N}} t^{N}|u(t, x, y)|^{p} \frac{\mathrm{d} t}{t} \mathrm{~d} x \frac{\mathrm{d} y}{t}\right)^{\frac{1}{p}}<+\infty
$$

Moreover, the weighted edge type $\mathcal{L}_{p}$-spaces with weight $\gamma \in \mathbb{R}$ are denoted by $\mathcal{L}_{p}^{\gamma}\left(\mathbb{R}_{+}^{N}\right.$, $\left.\frac{\mathrm{d} t}{t} \mathrm{~d} x \frac{\mathrm{d} y}{t}\right)$, which include functions $u(t, x, y)$ such that

$$
\|u\|_{\mathcal{L}_{p}^{\gamma}}=\left(\int_{\mathbb{R}_{+}^{N}} t^{N}\left|t^{-\gamma} u(t, x, y)\right|^{p} \frac{\mathrm{d} t}{t} \mathrm{~d} x \frac{\mathrm{d} y}{t}\right)^{\frac{1}{p}}<+\infty .
$$

The edge type weighted $p$-Sobolev spaces (cf. [8]) can be defined for all $1 \leq p<+\infty$ as follows.

Definition 2.4 Taking $\gamma \in \mathbb{R}, m \in \mathbb{N}$, and $N=1+n+q$, the edge type weighted $p$-Sobolev spaces are defined as

$$
\mathcal{H}_{p}^{m, \gamma}\left(\mathbb{R}_{+}^{N}\right):=\left\{u \in \mathcal{D}^{\prime}\left(\mathbb{R}_{+}^{N}\right):\left(t \partial_{t}\right)^{k} \partial_{x}^{\alpha}\left(t \partial_{y}\right)^{\beta} u \in \mathcal{L}_{p}^{\gamma}\left(\mathbb{R}_{+}^{N}, \frac{\mathrm{d} t}{t} \mathrm{~d} x \frac{\mathrm{d} y}{t}\right)\right\}
$$

for $k \in \mathbb{N}$, multi-indexes $\alpha \in \mathbb{N}^{n}, \beta \in \mathbb{N}^{q}$, and $k+|\alpha|+|\beta| \leq m$.

The edge type $p$-Sobolev spaces $\mathcal{H}_{p}^{m, \gamma}\left(\mathbb{R}_{+}^{N}\right)$ are Banach spaces with the norm

$$
\|u\|_{\mathcal{H}_{p}^{m, \gamma}\left(\mathbb{R}_{+}^{N}\right)}=\sum_{k+|\alpha|+|\beta| \leq m}\left(\int_{\mathbb{R}_{+}^{N}} t^{N}\left|t^{-\gamma}\left(t \partial_{t}\right)^{k} \partial_{x}^{\alpha}\left(t \partial_{y}\right)^{\beta} u(t, x, y)\right|^{p} \frac{\mathrm{d} t}{t} \mathrm{~d} x \frac{\mathrm{d} y}{t}\right)^{\frac{1}{p}}
$$

Finally, a corner can be defined as (cf. [9])

$$
E^{\Delta}=\left(\overline{\mathbb{R}}_{+} \times X^{\Delta}\right) /\left(\{0\} \times X^{\Delta}\right),
$$

where $X^{\Delta}$ is a cone. Then the corresponding stretched corner will be $\mathbb{E}^{\Delta}:=[0, r) \times X \times$ $[0, t), t, r \in \mathbb{R}_{+}$with the boundary $\{0\} \times X \times\{0\}$. Thus, under the local coordinates, the open stretched corner is $\mathbb{R}_{+} \times \mathbb{R}^{n} \times \mathbb{R}_{+}$. The typical degenerate differential operator on the open stretched corner $\mathbb{R}_{+} \times \mathbb{R}^{n} \times \mathbb{R}_{+}$will be

$$
(r t)^{-v} \sum_{j+k \leq \nu} a_{j k}(r, t)\left(r \partial_{r}\right)^{j}\left(r t \partial_{t}\right)^{k}=(r t)^{-v} A_{\mathbb{E}^{\Delta}}
$$

with coefficients $a_{j k}(r, t) \in C^{\infty}\left(\overline{\mathbb{R}}_{+}\right.$, Diff $\left.{ }^{\nu-j-k}\left(\mathbb{R}^{n}\right)\right)$ and $A_{\mathbb{E}^{\Delta}}$ is called a degenerate corner operator. Indeed, we have the following Riemannian metric on the corner $\mathbb{R}_{+} \times \mathbb{R}^{n} \times \mathbb{R}_{+}$:

$$
g:=\mathrm{d} t^{2}+t^{2}\left(\mathrm{~d} r^{2}+r^{2} \mathrm{~d} x^{2}\right)=(r t)^{2}\left[\left(\frac{\mathrm{d} t}{r t}\right)^{2}+\left(\frac{\mathrm{d} r}{r}\right)^{2}+\mathrm{d} x^{2}\right] .
$$

Then the corner type gradient operator will be $\mathrm{D}_{\text {cor }}:=\left(r \partial_{r}, \partial_{x_{1}}, \partial_{x_{2}}, \ldots, \partial_{x_{n}}, r t \partial_{t}\right)$.

Further, we give the definition of corner type weighted $\mathfrak{L}_{p}$-spaces as follows. 
Definition 2.5 Let $(r, x, t) \in \mathbb{R}_{+} \times \mathbb{R}^{n} \times \mathbb{R}_{+}$, weight data $\gamma_{1}, \gamma_{2} \in \mathbb{R}$, and $1 \leq p<+\infty$. Then $\mathfrak{L}_{p}^{\gamma_{1}, \gamma_{2}}\left(\mathbb{R}_{+} \times \mathbb{R}^{n} \times \mathbb{R}_{+}, \frac{\mathrm{d} r}{r} \mathrm{~d} x \frac{\mathrm{d} t}{r t}\right)$ denote the spaces of all $u(r, x, t) \in \mathcal{D}^{\prime}\left(\mathbb{R}_{+} \times \mathbb{R}^{n} \times \mathbb{R}_{+}\right)$such that

$$
\|u\|_{\mathfrak{L}_{p}^{\gamma_{1}, \gamma_{2}}}=\left(\int_{\mathbb{R}_{+} \times \mathbb{R}^{n} \times \mathbb{R}_{+}}\left|r^{\frac{N}{p}-\gamma_{1}} t^{\frac{N}{p}-\gamma_{2}} u(r, x, t)\right|^{p} \frac{\mathrm{d} r}{r} \mathrm{~d} x \frac{\mathrm{d} t}{r t}\right)^{\frac{1}{p}}<+\infty .
$$

From the weighted $\mathfrak{L}_{p}^{\gamma_{1}, \gamma_{2}}$-spaces, we can define the following weighted $p$-Sobolev spaces over stretched corner $\mathbb{R}_{+} \times \mathbb{R}^{n} \times \mathbb{R}_{+}$(cf. [9]).

Definition 2.6 Given $\gamma_{1}, \gamma_{2} \in \mathbb{R}, m \in \mathbb{N}, 1 \leq p<+\infty$, and $N=n+2$, the corner type weighted $p$-Sobolev spaces can be defined by

$$
\begin{aligned}
\mathbb{H}_{p}^{m,\left(\gamma_{1}, \gamma_{2}\right)}\left(\mathbb{R}_{+} \times \mathbb{R}^{n} \times \mathbb{R}_{+}\right) \\
=\left\{u \in \mathcal{D}^{\prime}\left(\mathbb{R}_{+} \times \mathbb{R}^{n} \times \mathbb{R}_{+}\right):\right. \\
\left.\quad\left(r \partial_{r}\right)^{l} \partial_{x}^{\alpha}\left(r t \partial_{t}\right)^{k} u(r, x, t) \in \mathfrak{L}_{p}^{\gamma_{1}, \gamma_{2}}\left(\mathbb{R}_{+} \times \mathbb{R}^{n} \times \mathbb{R}_{+}, \frac{\mathrm{d} r}{r} \mathrm{~d} x \frac{\mathrm{d} t}{r t}\right)\right\}
\end{aligned}
$$

for $k, l \in \mathbb{N}$, multi-index $\alpha \in \mathbb{N}^{n}$, and $k+|\alpha|+l \leq m$.

It can be proved that $\mathbb{H}_{p}^{m,\left(\gamma_{1}, \gamma_{2}\right)}\left(\mathbb{R}_{+} \times \mathbb{R}^{n} \times \mathbb{R}_{+}\right)$are Banach spaces equipped with the norm

$$
\begin{aligned}
& \|u\|_{\mathbb{H}_{p}^{m,\left(\gamma_{1}, \gamma_{2}\right)}}\left(\mathbb{R}_{+} \times \mathbb{R}^{n} \times \mathbb{R}_{+}\right) \\
& \quad=\sum_{k+|\alpha|+l \leq m}\left(\int_{\mathbb{R}_{+} \times \mathbb{R}^{n} \times \mathbb{R}_{+}}\left|r^{\frac{N}{p}-\gamma_{1}} t^{\frac{N}{p}-\gamma_{2}}\left(r \partial_{r}\right)^{l} \partial_{x}^{\alpha}\left(r t \partial_{t}\right)^{k} u(r, x, t)\right|^{p} \frac{\mathrm{d} r}{r} \mathrm{~d} x \frac{\mathrm{d} t}{r t}\right)^{\frac{1}{p}} .
\end{aligned}
$$

\section{Proof of Theorem 1.1}

In this section, we give the proof of Theorem 1.1.

Proof Let $\sigma_{i}=1+s\left(1-\frac{1}{p_{i}}\right) \geq 1$ and $v_{i}(t, x)=\left(t^{\frac{n+1}{s}-\gamma}|u(t, x)|\right)^{\sigma_{i}}$ for $0 \leq i \leq n$. From $\frac{1}{s}=$ $\frac{1}{n+1}\left(\sum_{i=0}^{n} \frac{1}{p_{i}}-1\right)$, then it holds that $\sum_{i=0}^{n} \sigma_{i}=n s$.

Since $u(t, x) \in C_{0}^{\infty}\left(\mathbb{R}_{+}^{n+1}\right)$, then we have, for $i=0$ and $t>0$,

$$
2 v_{0}(t, x)=\int_{0}^{t} \tau \frac{\partial v_{0}(\tau, x)}{\partial \tau} \frac{\mathrm{d} \tau}{\tau}-\int_{t}^{+\infty} \tau \frac{\partial v_{0}(\tau, x)}{\partial \tau} \frac{\mathrm{d} \tau}{\tau} .
$$

Thus

$$
\begin{aligned}
\left|2 v_{0}(t, x)\right| & \leq \int_{0}^{t}\left|\tau \frac{\partial v_{0}(\tau, x)}{\partial \tau}\right| \frac{\mathrm{d} \tau}{\tau}+\int_{t}^{+\infty}\left|\tau \frac{\partial v_{0}(\tau, x)}{\partial \tau}\right| \frac{\mathrm{d} \tau}{\tau} \\
& =: \int_{0}^{+\infty}\left|\left(t \partial_{t}\right) v_{0}(t, x)\right| \frac{\mathrm{d} t}{t} .
\end{aligned}
$$

Analogously, for $1 \leq i \leq n$,

$$
\left|2 v_{i}(t, x)\right| \leq \int_{-\infty}^{+\infty}\left|\frac{\partial v_{i}(t, x)}{\partial x_{i}}\right| \mathrm{d} x_{i} .
$$


After multiplying the $n+1$ inequalities above, we have

$$
2^{\frac{n+1}{n}}\left(t^{\frac{n+1}{s}-\gamma}|u(t, x)|\right)^{s} \leq\left(\int_{0}^{+\infty}\left|\left(t \partial_{t}\right) v_{0}(t, x)\right| \frac{\mathrm{d} t}{t}\right)^{\frac{1}{n}} \prod_{i=1}^{n}\left(\int_{-\infty}^{+\infty}\left|\frac{\partial v_{i}(t, x)}{\partial x_{i}}\right| \mathrm{d} x_{i}\right)^{\frac{1}{n}}
$$

Now integrating the inequality above over the interval $(0,+\infty)$ with respect to $\frac{\mathrm{d} t}{t}$ and using Hölder's inequality, we obtain

$$
\begin{aligned}
& 2^{\frac{n+1}{n}} \int_{0}^{+\infty}\left(t^{\frac{n+1}{s}-\gamma}|u(t, x)|\right)^{s} \frac{\mathrm{d} t}{t} \\
& \quad \leq\left(\int_{0}^{+\infty}\left|\left(t \partial_{t}\right) v_{0}(t, x)\right| \frac{\mathrm{d} t}{t}\right)^{\frac{1}{n}} \prod_{i=1}^{n}\left(\int_{0}^{+\infty} \int_{-\infty}^{+\infty}\left|\frac{\partial v_{i}(t, x)}{\partial x_{i}}\right| \mathrm{d} x_{i} \frac{\mathrm{d} t}{t}\right)^{\frac{1}{n}} .
\end{aligned}
$$

Then integrating above inequality again over the interval $(-\infty,+\infty)$ with respect to $x_{1}, x_{2}, \ldots, x_{n}$ and using Hölder's inequality respectively, we can deduce that

$$
\begin{aligned}
& 2^{\frac{n+1}{n}} \int_{\mathbb{R}^{n}} \int_{\mathbb{R}_{+}}\left(t^{\frac{n+1}{s}-\gamma}|u(t, x)|\right)^{s} \frac{\mathrm{d} t}{t} \mathrm{~d} x \\
& \quad \leq\left(\int_{\mathbb{R}^{n}} \int_{\mathbb{R}_{+}}\left|\left(t \partial_{t}\right) v_{0}(t, x)\right| \frac{\mathrm{d} t}{t} \mathrm{~d} x\right)^{\frac{1}{n}} \prod_{i=1}^{n}\left(\int_{\mathbb{R}^{n}} \int_{\mathbb{R}_{+}}\left|\frac{\partial v_{i}(t, x)}{\partial x_{i}}\right| \frac{\mathrm{d} t}{t} \mathrm{~d} x\right)^{\frac{1}{n}} .
\end{aligned}
$$

For $1 \leq i \leq n,\left|\partial_{x_{i}}\right| u(t, x)||=\left|\partial_{x_{i}}(u \bar{u})^{\frac{1}{2}}\right|=\frac{1}{2}\left|(\bar{u} u)^{-\frac{1}{2}}\left(\bar{u} \partial_{x_{i}} u+u \partial_{x_{i}} \bar{u}\right)\right| \leq \frac{1}{2}|u|^{-1}\left(\left|\bar{u} \partial_{x_{i}} u\right|+\right.$ $\left.\left|u \partial_{x_{i}} \bar{u}\right|\right) \leq\left|\partial_{x_{i}} u(t, x)\right|$. Thus we obtain

$$
\left|\partial_{x_{i}} v_{i}(t, x)\right| \leq \sigma_{i}\left(t^{\frac{n+1}{s}-\gamma}|u(t, x)|\right)^{\sigma_{i}-1} t^{\frac{n+1}{s}-\gamma}\left|\partial_{x_{i}} u(t, x)\right|
$$

Similarly, $\left|\left(t \partial_{t}\right)\right| u(t, x)|\leq|\left(t \partial_{t}\right) u(t, x) \mid$, then we have

$$
\begin{aligned}
& \left|\left(t \partial_{t}\right) v_{0}(t, x)\right| \\
& \quad \leq \sigma_{0}\left(t^{\frac{n+1}{s}-\gamma}|u(t, x)|\right)^{\sigma_{0}-1}\left[\left|\frac{n+1}{s}-\gamma\right| t^{\frac{n+1}{s}-\gamma}|u(t, x)|+t^{\frac{n+1}{s}-\gamma}\left|\left(t \partial_{t}\right) u(t, x)\right|\right] .
\end{aligned}
$$

Replace the corresponding parts of (3.1) by (3.2) and (3.3), we derive that

$$
\begin{aligned}
2^{\frac{n+1}{n}} & \int_{\mathbb{R}^{n}} \int_{\mathbb{R}_{+}}\left(t^{\frac{n+1}{s}-\gamma}|u(t, x)|\right)^{s} \frac{\mathrm{d} t}{t} \mathrm{~d} x \\
\leq & {\left[\sigma_{0}\left|\frac{n+1}{s}-\gamma\right| \int_{\mathbb{R}^{n}} \int_{\mathbb{R}_{+}}\left(t^{\frac{n+1}{s}-\gamma}|u(t, x)|\right)^{\sigma_{0}-1}\left(t^{\frac{n+1}{s}-\gamma}|u(t, x)|\right) \frac{\mathrm{d} t}{t} \mathrm{~d} x\right.} \\
& \left.+\sigma_{0} \int_{\mathbb{R}^{n}} \int_{\mathbb{R}_{+}}\left(t^{\frac{n+1}{s}-\gamma}|u(t, x)|\right)^{\sigma_{0}-1}\left(t^{\frac{n+1}{s}-\gamma}\left|\left(t \partial_{t}\right) u(t, x)\right|\right) \frac{\mathrm{d} t}{t} \mathrm{~d} x\right]^{\frac{1}{n}} \\
& \times \prod_{i=1}^{n}\left(\int_{\mathbb{R}^{n}} \int_{\mathbb{R}_{+}} \sigma_{i}\left(t^{\frac{n+1}{s}-\gamma}|u(t, x)|\right)^{\sigma_{i}-1} t^{\frac{n+1}{s}-\gamma}\left|\partial_{x_{i}} u(t, x)\right| \frac{\mathrm{d} t}{t} \mathrm{~d} x\right)^{\frac{1}{n}} \\
=: & \left(I_{1}+I_{2}\right)^{\frac{1}{n}} \cdot I_{3} .
\end{aligned}
$$


Case 1: $p_{i}>1$ for $0 \leq i \leq n$.

If $p_{i}^{\prime}$ satisfies $\frac{1}{p_{i}^{\prime}}+\frac{1}{p_{i}}=1$, then $\left(\sigma_{i}-1\right) p_{i}^{\prime}=s\left(1-\frac{1}{p_{i}}\right) p_{i}^{\prime}=s$ for $0 \leq i \leq n$. By Hölder's inequality, we can acquire that

$$
\begin{aligned}
& I_{1}=\sigma_{0}\left|\frac{n+1}{s}-\gamma\right| \int_{\mathbb{R}^{n}} \int_{\mathbb{R}_{+}}\left(t^{\frac{n+1}{s}-\gamma}|u(t, x)|\right)^{\sigma_{0}-1}\left(t^{\frac{n+1}{s}-\gamma}|u(t, x)|\right) \frac{\mathrm{d} t}{t} \mathrm{~d} x \\
& \leq \sigma_{0}\left|\frac{n+1}{s}-\gamma\right|\|u\|_{L_{s}^{\gamma}}^{\frac{s}{p_{0}^{\prime}}}\|u\|_{L_{p_{0}}^{-\left(\frac{n+1}{s}-\gamma-\frac{n+1}{p_{0}}\right)}}, \\
& I_{2}=\sigma_{0} \int_{\mathbb{R}^{n}} \int_{\mathbb{R}_{+}}\left(t^{\frac{n+1}{s}-\gamma}|u(t, x)|\right)^{\sigma_{0}-1}\left(t^{\frac{n+1}{s}-\gamma}\left|\left(t \partial_{t}\right) u(t, x)\right|\right) \frac{\mathrm{d} t}{t} \mathrm{~d} x \\
& \leq \sigma_{0}\|u\|_{L_{s}^{\gamma}}^{\frac{s}{p_{0}^{\prime}}}\left\|\left(t \partial_{t}\right) u\right\|_{L_{p_{0}}^{-\left(\frac{n+1}{s}-\gamma-\frac{n+1}{p_{0}}\right)}}, \\
& I_{3}=\prod_{i=1}^{n}\left(\int_{\mathbb{R}^{n}} \int_{\mathbb{R}_{+}} \sigma_{i}\left(t^{\frac{n+1}{s}-\gamma}|u(t, x)|\right)^{\sigma_{i}-1} t^{\frac{n+1}{s}-\gamma}\left|\partial_{x_{i}} u(t, x)\right| \frac{\mathrm{d} t}{t} \mathrm{~d} x\right)^{\frac{1}{n}} \\
& \leq \prod_{i=1}^{n} \sigma_{i}^{\frac{1}{n}}\|u\|_{L_{s}^{\gamma}}^{\frac{s}{n p_{i}^{\prime}}}\left\|\partial_{x_{i}} u\right\|_{L_{p_{i}}}^{\frac{1}{n}}{ }_{\left(\frac{n+1}{s}-\gamma-\frac{n+1}{p_{i}}\right)} .
\end{aligned}
$$

Returning to (3.4) and setting $\gamma_{i}^{*}=-\left(\frac{n+1}{s}-\gamma-\frac{n+1}{p_{i}}\right)$ for $0 \leq i \leq n$, we get

$$
\begin{aligned}
2^{\frac{n+1}{n}}\|u\|_{L_{s}^{\gamma}}^{s} \leq & \left(\sigma_{0}\left|\frac{n+1}{s}-\gamma\right|\|u\|_{L_{p_{0}}^{\gamma_{0}^{*}}}+\sigma_{0}\left\|\left(t \partial_{t}\right) u\right\|_{L_{p_{0}}^{\gamma_{0}^{*}}}\right)^{\frac{1}{n}} \prod_{i=0}^{n}\|u\|_{L_{s}^{\gamma}}^{\frac{s}{n p_{i}^{\prime}}} \\
& \times \prod_{i=1}^{n} \sigma_{i}^{\frac{1}{n}}\left\|\partial_{x_{i}} u\right\|_{L_{p_{i}}^{\gamma_{*}^{*}}}^{\frac{1}{n}}
\end{aligned}
$$

In view of $\frac{n+1}{s}+1=\sum_{i=0}^{n} \frac{1}{p_{i}}$, we deduce $\sum_{i=0}^{n} \frac{s}{n p_{i}^{\prime}}=\frac{s}{n}\left(n+1-\sum_{i=0}^{n} \frac{1}{p_{i}}\right)=s-\frac{n+1}{n}$. According to (3.5), we find that

$$
2^{\frac{n+1}{n}}\|u\|_{L_{s}^{\frac{n+1}{\gamma}}}^{\frac{n+1}{\gamma}} \leq\left(\sigma_{0}\left|\frac{n+1}{s}-\gamma\right|\|u\|_{L_{p_{0}}^{\gamma_{0}^{*}}}+\sigma_{0}\left\|\left(t \partial_{t}\right) u\right\|_{L_{p_{0}}^{\gamma_{0}^{*}}}\right)^{\frac{1}{n}}\left(\prod_{i=1}^{n} \sigma_{i}\left\|\partial_{x_{i}} u\right\|_{L_{p_{i}}^{\gamma_{i}^{*}}}\right)^{\frac{1}{n}} .
$$

That means

$$
\begin{aligned}
\|u\|_{L_{s}^{\gamma}} & \leq \frac{1}{2}\left(\sigma_{0}\left|\frac{n+1}{s}-\gamma\right|\|u\|_{L_{p_{0}}^{\gamma_{0}^{*}}}+\sigma_{0}\left\|\left(t \partial_{t}\right) u\right\|_{L_{p_{0}}^{\gamma_{0}^{*}}}\right)^{\frac{1}{n+1}} \prod_{i=1}^{n} \sigma_{i}^{\frac{1}{n+1}}\left\|\partial_{x_{i}} u\right\|_{L_{p_{i}}^{\gamma_{i}^{*}}}^{\frac{1}{n+1}} \\
& \leq \frac{1}{2}\left[\left(\sigma_{0}\left|\frac{n+1}{s}-\gamma\right|\right)^{\frac{1}{n+1}}\|u\|_{L_{p_{0}}^{\gamma_{*}^{*}}}^{\frac{1}{n+1}}+\sigma_{0}^{\frac{1}{n+1}}\left\|\left(t \partial_{t}\right) u\right\|_{L_{p_{0}}^{\gamma_{0}^{*}}}^{\frac{1}{n+1}} \prod_{i=1}^{n} \sigma_{i}^{\frac{1}{n+1}}\left\|\partial_{x_{i}} u\right\|_{\substack{\frac{1}{n+1} \\
L_{p_{i}^{*}}^{\gamma_{i}^{*}}}}\right.
\end{aligned}
$$

Set $c_{01}=\frac{1}{2}\left(\sigma_{0}\left|\frac{n+1}{s}-\gamma\right|\right)^{\frac{1}{n+1}}=\frac{1}{2}\left[\left(1+\frac{s\left(p_{0}-1\right)}{p_{0}}\right)\left|\frac{n+1}{s}-\gamma\right|\right]^{\frac{1}{n+1}}, c_{02}=\frac{1}{2} \sigma_{0}^{\frac{1}{n+1}}=\frac{1}{2}\left(1+\frac{s\left(p_{0}-1\right)}{p_{0}}\right)^{\frac{1}{n+1}}$, and $c_{i}=\sigma_{i}^{\frac{1}{n+1}}=\left(1+\frac{s\left(p_{i}-1\right)}{p_{i}}\right)^{\frac{1}{n+1}}$ for $1 \leq i \leq n$. As a consequence,

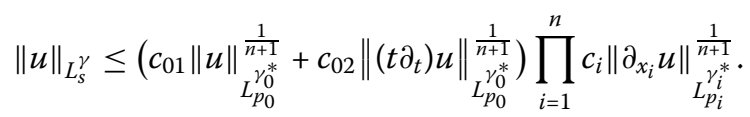


Case 2: There exists at least one $p_{i} \in\left\{p_{0}, p_{1}, \ldots, p_{n}\right\}$ such that $p_{i}=1$.

Without loss of generality, set $p_{0}, p_{1}, p_{2}, \ldots, p_{i_{0}}=1$ and $p_{i_{0}+1}, \ldots, p_{n}>1$. We deduce that $\sigma_{i}=1\left(0 \leq i \leq i_{0}\right), \sigma_{i}>1\left(i_{0}+1 \leq i \leq n\right)$, and $\frac{1}{s}=\frac{1}{n+1}\left(i_{0}+\sum_{i=i_{0}+1}^{n} \frac{1}{p_{i}}\right)$. Thus inequality (3.4) becomes

$$
\begin{aligned}
2^{\frac{n+1}{n}} & \int_{\mathbb{R}^{n}} \int_{\mathbb{R}_{+}}\left(t^{\frac{n+1}{s}-\gamma}|u(t, x)|\right)^{s} \frac{\mathrm{d} t}{t} \mathrm{~d} x \\
\leq & {\left[\left|\frac{n+1}{s}-\gamma\right| \int_{\mathbb{R}^{n}} \int_{\mathbb{R}_{+}} t^{\frac{n+1}{s}-\gamma}|u(t, x)| \frac{\mathrm{d} t}{t} \mathrm{~d} x+\int_{\mathbb{R}^{n}} \int_{\mathbb{R}_{+}}\left(t^{\frac{n+1}{s}-\gamma}\left|\left(t \partial_{t}\right) u(t, x)\right|\right) \frac{\mathrm{d} t}{t} \mathrm{~d} x\right]^{\frac{1}{n}} } \\
& \times\left(\prod_{i=1}^{i_{0}} \int_{\mathbb{R}^{n}} \int_{\mathbb{R}_{+}} t^{\frac{n+1}{s}-\gamma}\left|\partial_{x_{i}} u(t, x)\right| \frac{\mathrm{d} t}{t} \mathrm{~d} x\right)^{\frac{1}{n}} \\
& \times\left(\prod_{i=i_{0}+1}^{n} \int_{\mathbb{R}^{n}} \int_{\mathbb{R}_{+}} \sigma_{i}\left(t^{\frac{n+1}{s}-\gamma}|u(t, x)|\right)^{\sigma_{i}-1} t^{\frac{n+1}{s}-\gamma}\left|\partial_{x_{i}} u(t, x)\right| \frac{\mathrm{d} t}{t} \mathrm{~d} x\right)^{\frac{1}{n}} \\
= & \left(\hat{I}_{1}+\hat{I}_{2}\right)^{\frac{1}{n}} \hat{I}_{3} \hat{I}_{4} .
\end{aligned}
$$

For $\hat{I}_{4}$, setting $\frac{1}{p_{i}}+\frac{1}{p_{i}^{\prime}}=1\left(i_{0}+1 \leq i \leq n\right)$ and using Hölder's inequality again, we have

$$
\hat{I}_{4} \leq \prod_{i=i_{0}+1}^{n}\|u\|_{L_{s}^{\gamma}}^{\frac{s}{n p_{i}^{\prime}}} \prod_{i=i_{0}+1}^{n} \sigma_{i}^{\frac{1}{n}}\left\|\partial_{x_{i}} u\right\|_{L_{p_{i}}}^{\frac{1}{n}}
$$

Further, it follows from $\sum_{i=i_{0}+1}^{n} \frac{s}{n p_{i}^{\prime}}=\frac{s}{n}\left(n-i_{0}-\sum_{i=i_{0}+1}^{n} \frac{1}{p_{i}}\right)=s-\frac{n+1}{n}$ that

$$
\begin{aligned}
2^{\frac{n+1}{n}}\|u\|_{L_{s}^{\gamma}}^{\frac{n+1}{n}} \leq & \left(\left|\frac{n+1}{s}-\gamma\right|\|u\|_{L_{1}^{-\left(\frac{n+1}{s}-\gamma-n-1\right)}}+\left\|\left(t \partial_{t}\right) u\right\|_{L_{1}^{-\left(\frac{n+1}{s}-\gamma-n-1\right)}}\right)^{\frac{1}{n}} \\
& \times\left(\prod_{i=1}^{i_{0}}\left\|\partial_{x_{i}} u\right\|_{L_{1}^{-\left(\frac{n+1}{s}-\gamma-n-1\right)}}\right)^{\frac{1}{n}}\left(\prod_{i=i_{0}+1}^{n} \sigma_{i}\left\|\partial_{x_{i}} u\right\|_{L_{p_{i}}\left(\frac{n+1}{s}-\gamma-\frac{n+1}{p_{i}}\right)}\right)^{\frac{1}{n}} .
\end{aligned}
$$

Hence we can acquire that

$$
\begin{aligned}
& \|u\|_{L_{s}^{\gamma}} \leq \frac{1}{2}\left(\left|\frac{n+1}{s}-\gamma\right|^{\frac{1}{n+1}}\|u\|_{L_{1}^{-\left(\frac{n+1}{s}-\gamma-n-1\right)}}^{\frac{1}{n+1}}+\left\|\left(t \partial_{t}\right) u\right\|_{L_{1}^{\left(-\frac{n+1}{s}-\gamma-n-1\right)}}^{\frac{1}{n+1}}\right) \\
& \times\left(\prod_{i=1}^{i_{0}}\left\|\partial_{x_{i}} u\right\|_{L_{1}^{-\left(\frac{n+1}{s}-\gamma-n-1\right)}}\right)^{\frac{1}{n+1}} \prod_{i=i_{0}+1}^{n} \sigma_{i}^{\frac{1}{n+1}}\left\|\partial_{x_{i}} u\right\|_{L_{p_{i}}^{-\left(\frac{n+1}{s}-\gamma-\frac{n+1}{p_{i}}\right)}}^{\frac{1}{n+1}}
\end{aligned}
$$

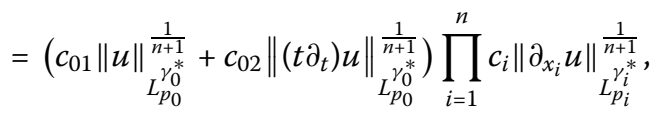

where $c_{01}, c_{02}, c_{i}(1 \leq i \leq n)$ and $\gamma_{i}^{*}(0 \leq i \leq n)$ are the same as those in (3.7). Theorem 1.1 is proved. 


\section{Proofs of Theorem 1.3 and Theorem 1.5}

\subsection{Proof of Theorem 1.3}

Proof Let $\sigma_{i}=1+s\left(1-\frac{1}{p_{i}}\right) \geq 1$ and $v_{i}(t, x)=\left(t^{\frac{n+q+1}{s}-\gamma}|u(t, x)|\right)^{\sigma_{i}}$ for $0 \leq i \leq n+q$. Since $\frac{1}{s}=\frac{1}{n+q+1}\left(\sum_{i=0}^{n+q} \frac{1}{p_{i}}-1\right)$, then we have $\sum_{i=0}^{n+q} \sigma_{i}=(n+q) s$.

For $u(t, x, y) \in C_{0}^{\infty}\left(\mathbb{R}_{+}^{n+q+1}\right)$, it holds that

$$
2 v_{0}(t, x, y)=\int_{0}^{t} \tau \frac{\partial v_{0}(\tau, x, y)}{\partial \tau} \frac{\mathrm{d} \tau}{\tau}-\int_{t}^{+\infty} \tau \frac{\partial v_{0}(\tau, x, y)}{\partial \tau} \frac{\mathrm{d} \tau}{\tau}
$$

Thus

$$
\begin{aligned}
\left|2 v_{0}(t, x, y)\right| & \leq \int_{0}^{t}\left|\tau \frac{\partial v_{0}(\tau, x, y)}{\partial \tau}\right| \frac{\mathrm{d} \tau}{\tau}+\int_{t}^{+\infty}\left|\tau \frac{\partial v_{0}(\tau, x, y)}{\partial \tau}\right| \frac{\mathrm{d} \tau}{\tau} \\
& \leq \int_{0}^{+\infty}\left|\left(t \partial_{t}\right) v_{0}(t, x, y)\right| \frac{\mathrm{d} t}{t}
\end{aligned}
$$

Also, for $1 \leq i \leq n$, one has

$$
\begin{aligned}
\left|2 v_{i}(t, x, y)\right| \leq & \int_{-\infty}^{x_{i}}\left|\frac{\partial v_{i}\left(t, x_{1}, x_{2}, \ldots, x_{i-1}, \hat{x}_{i}, x_{i+1}, \ldots, x_{n}, y\right)}{\partial \hat{x}_{i}}\right| \mathrm{d} \hat{x}_{i} \\
& +\int_{x_{i}}^{+\infty}\left|\frac{\partial v_{i}\left(t, x_{1}, x_{2}, \ldots, x_{i-1}, \hat{x}_{i}, x_{i+1}, \ldots, x_{n}, y\right)}{\partial \hat{x}_{i}}\right| \mathrm{d} \hat{x}_{i} \\
\leq & \int_{-\infty}^{+\infty}\left|\partial_{x_{i}} v_{i}(t, x, y)\right| \mathrm{d} x_{i},
\end{aligned}
$$

where $y=\left(y_{n+1}, y_{n+2}, \ldots, y_{n+q}\right)$. Similarly, for $n+1 \leq i \leq n+q$, we derive

$$
\begin{aligned}
\left|2 v_{i}(t, x, y)\right| \leq & \int_{-\infty}^{y_{i}}\left|\frac{\partial v_{i}\left(t, x, y_{n+1}, \ldots, y_{i-1}, \hat{y}_{i}, y_{i+1}, \ldots, y_{n+q}\right)}{\partial \hat{y}_{i}}\right| \mathrm{d} \hat{y}_{i} \\
& +\int_{y_{i}}^{+\infty}\left|\frac{\partial v_{i}\left(t, x, y_{n+1}, \ldots, y_{i-1}, \hat{y}_{i}, y_{i+1}, \ldots, y_{n+q}\right)}{\partial \hat{y}_{i}}\right| \mathrm{d} \hat{y}_{i} \\
= & \int_{-\infty}^{+\infty}\left|\left(t \partial_{\hat{y}_{i}}\right) v_{i}\left(t, x, y_{n+1}, \ldots, y_{i-1}, \hat{y}_{i}, y_{i+1}, \ldots, y_{n+q}\right)\right| \frac{\mathrm{d} \hat{y}_{i}}{t} \\
= & : \int_{-\infty}^{+\infty}\left|\left(t \partial_{y_{i}}\right) v_{i}(t, x, y)\right| \frac{\mathrm{d} y_{i}}{t},
\end{aligned}
$$

where $x=\left(x_{1}, x_{2}, \ldots, x_{n}\right) \in \mathbb{R}^{n}$. By multiplying the $n+q+1$ inequalities above, we have

$$
\begin{aligned}
\prod_{i=0}^{n+q}\left|2 v_{i}(t, x)\right|= & 2^{n+q+1}\left(t^{\frac{n+q+1}{s}-\gamma}|u(t, x, y)|\right)^{\sum_{i=0}^{n+q} \sigma_{i}} \\
= & 2^{n+q+1}\left(t^{\frac{n+q+1}{s}-\gamma}|u(t, x, y)|\right)^{s(n+q)} \\
\leq & \int_{0}^{+\infty}\left|\left(t \partial_{t}\right) v_{0}(t, x, y)\right| \frac{\mathrm{d} t}{t} \prod_{i=1}^{n} \int_{-\infty}^{+\infty}\left|\partial_{x_{i}} v_{i}(t, x, y)\right| \mathrm{d} x_{i} \\
& \times \prod_{i=n+1}^{n+q} \int_{-\infty}^{+\infty}\left|\left(t \partial_{y_{i}}\right) v_{i}(t, x, y)\right| \frac{\mathrm{d} y_{i}}{t} .
\end{aligned}
$$


That implies

$$
\begin{aligned}
2^{\frac{n+q+1}{n+q}}\left(t^{\frac{n+q+1}{s}-\gamma}|u(t, x, y)|\right)^{s} \leq & \left(\int_{0}^{+\infty}\left|\left(t \partial_{t}\right) v_{0}(t, x, y)\right| \frac{\mathrm{d} t}{t}\right)^{\frac{1}{n+q}} \\
& \times \prod_{i=1}^{n}\left(\int_{-\infty}^{+\infty}\left|\partial_{x_{i}} v_{i}(t, x, y)\right| \mathrm{d} x_{i}\right)^{\frac{1}{n+q}} \\
& \times \prod_{i=n+1}^{n+q}\left(\int_{-\infty}^{+\infty}\left|\left(t \partial_{y_{i}}\right) v_{i}(t, x, y)\right| \frac{\mathrm{d} y_{i}}{t}\right)^{\frac{1}{n+q}}
\end{aligned}
$$

Now integrating these inequalities over the interval $(0,+\infty)$ with respect to $\frac{\mathrm{d} t}{t}$ and using Hölder inequality will lead to

$$
\begin{aligned}
2^{\frac{n+q+1}{n+q}} & \int_{0}^{+\infty}\left(t^{\frac{n+q+1}{s}-\gamma}|u(t, x, y)|\right)^{s} \frac{\mathrm{d} t}{t} \\
\leq & \left(\int_{0}^{+\infty}\left|\left(t \partial_{t}\right) v_{0}(t, x, y)\right| \frac{\mathrm{d} t}{t}\right)^{\frac{1}{n+q}} \prod_{i=1}^{n}\left(\int_{0}^{+\infty} \int_{-\infty}^{+\infty}\left|\partial_{x_{i}} v_{i}(t, x, y)\right| \mathrm{d} x_{i} \frac{\mathrm{d} t}{t}\right)^{\frac{1}{n+q}} \\
& \times \prod_{i=n+1}^{n+q}\left(\int_{0}^{+\infty} \int_{-\infty}^{+\infty}\left|\left(t \partial_{y_{i}}\right) v_{i}(t, x, y)\right| \frac{\mathrm{d} y_{i}}{t} \frac{\mathrm{d} t}{t}\right)^{\frac{1}{n+q}} .
\end{aligned}
$$

Then integrating over the interval $(-\infty,+\infty)$ with respect to $x_{1}, x_{2}, \ldots, x_{n}$ and $\frac{\mathrm{d} y_{n+1}}{t}, \ldots$, $\frac{\mathrm{d} y_{n+q}}{t}$ respectively and using Hölder's inequality again, we obtain by setting $\mathrm{d} \eta:=\frac{\mathrm{d} t}{t} \mathrm{~d} x \frac{\mathrm{d} y}{t}$ and $N=n+q+1$ that

$$
\begin{aligned}
2^{\frac{n+q+1}{n+1}} & \int_{\mathbb{R}_{+}^{N}}\left(t^{\frac{n+q+1}{s}-\gamma}|u(t, x, y)|\right)^{s} \mathrm{~d} \eta \\
\leq & \left(\int_{\mathbb{R}_{+}^{N}}\left|\left(t \partial_{t}\right) v_{0}(t, x, y)\right| \mathrm{d} \eta\right)^{\frac{1}{n+q}} \prod_{i=1}^{n}\left(\int_{\mathbb{R}_{+}^{N}}\left|\partial_{x_{i}} v_{i}(t, x, y)\right| \mathrm{d} \eta\right)^{\frac{1}{n+q}} \\
& \times \prod_{i=n+1}^{n+q}\left(\int_{\mathbb{R}_{+}^{N}}\left|\left(t \partial_{y_{i}}\right) v_{i}(t, x, y)\right| \mathrm{d} \eta\right)^{\frac{1}{n+q}}
\end{aligned}
$$

For $1 \leq i \leq n$, we acquire $\left|\partial_{x_{i}}\right| u(t, x, y)||=\left|\partial_{x_{i}}(u \bar{u})^{\frac{1}{2}}\right| \leq \frac{1}{2}|u|^{-1}\left(\left|\bar{u} \partial_{x_{i}} u\right|+\left|u \partial_{x_{i}} \bar{u}\right|\right) \leq$ $\left|\partial_{x_{i}} u(t, x, y)\right|$. Similar to this deduction, it holds that $\left|\left(t \partial_{t}\right)\right| u(t, x, y)|| \leq\left|\left(t \partial_{t}\right) u(t, x, y)\right|$ and $\left|\left(t \partial_{y_{i}}\right)\right| u(t, x, y)|| \leq\left|\left(t \partial_{y_{i}}\right) u(t, x, y)\right|$ for $n+1 \leq i \leq n+q$.

Consequently, for $1 \leq i \leq n$, we have

$$
\begin{aligned}
\left|\partial_{x_{i}} \nu_{i}(t, x, y)\right|= & \left|\partial_{x_{i}}\left(t^{\frac{n+q+1}{s}-\gamma}|u(t, x, y)|\right)^{\sigma_{i}}\right| \\
\leq & \sigma_{i}\left(t^{\frac{n+q+1}{s}-\gamma}|u(t, x, y)|\right)^{\sigma_{i}-1} t^{\frac{n+q+1}{s}-\gamma}\left|\partial_{x_{i}} u(t, x, y)\right| \\
\left|\left(t \partial_{t}\right) v_{0}(t, x, y)\right| \leq & \sigma_{0}\left(t^{\frac{n+q+1}{s}-\gamma}|u(t, x, y)|\right)^{\sigma_{0}-1}\left[\left|\frac{n+q+1}{s}-\gamma\right| t^{\frac{n+q+1}{s}-\gamma}|u(t, x, y)|\right. \\
& \left.+t^{\frac{n+q+1}{s}-\gamma}\left|\left(t \partial_{t}\right) u(t, x, y)\right|\right] .
\end{aligned}
$$


Also, for $n+1 \leq i \leq n+q$, we have

$$
\left|\left(t \partial_{y_{i}}\right) v_{i}(t, x, y)\right| \leq \sigma_{i}\left(t^{\frac{n+q+1}{s}-\gamma}|u(t, x, y)|\right)^{\sigma_{i}-1} t^{\frac{n+q+1}{s}-\gamma}\left|\left(t \partial_{y_{i}}\right) u(t, x, y)\right| .
$$

After rewriting the corresponding parts of (4.1) by (4.2), (4.3), and (4.4), we get

$$
\begin{aligned}
2^{\frac{n+q+1}{n+q}} & \int_{\mathbb{R}_{+}^{N}}\left(t^{\frac{n+q+1}{s}-\gamma}|u(t, x, y)|\right)^{s} \mathrm{~d} \eta \\
\leq & {\left[\sigma_{0}\left|\frac{n+q+1}{s}-\gamma\right| \int_{\mathbb{R}_{+}^{N}}\left(t^{\frac{n+q+1}{s}-\gamma}|u(t, x, y)|\right)^{\sigma_{0}-1}\left(t^{\frac{n+q+1}{s}-\gamma}|u(t, x, y)|\right) \mathrm{d} \eta\right.} \\
& \left.+\sigma_{0} \int_{\mathbb{R}_{+}^{N}}\left(t^{\frac{n+q+1}{s}-\gamma}|u(t, x, y)|\right)^{\sigma_{0}-1}\left(t^{\frac{n+q+1}{s}-\gamma}\left|\left(t \partial_{t}\right) u(t, x, y)\right|\right) \mathrm{d} \eta\right]^{\frac{1}{n+q}} \\
& \times \prod_{i=1}^{n}\left[\int_{\mathbb{R}_{+}^{N}} \sigma_{i}\left(t^{\frac{n+q+1}{s}-\gamma}|u(t, x, y)|\right)^{\sigma_{i}-1}\left(t^{\frac{n+q+1}{s}-\gamma}\left|\partial_{x_{i}} u(t, x, y)\right|\right) \mathrm{d} \eta\right]^{\frac{1}{n+q}} \\
& \times \prod_{i=n+1}^{n+q}\left[\int_{\mathbb{R}_{+}^{N}} \sigma_{i}\left(t^{\frac{n+q+1}{s}}-\gamma|u(t, x, y)|\right)^{\sigma_{i}-1}\left(t^{\frac{n+q+1}{s}-\gamma}\left|\left(t \partial_{y_{i}}\right) u(t, x, y)\right|\right) \mathrm{d} \eta\right]^{\frac{1}{n+q}} \\
=: & \left(I_{1}+I_{2}\right)^{\frac{1}{n}} \cdot I_{3} \cdot I_{4} .
\end{aligned}
$$

There are still two cases similar to the proof of Theorem 1.1, i.e., the case of $p_{i}>1$ for $0 \leq i \leq n+q$ and the case that there exists at least one $p_{i} \in\left\{p_{0}, p_{1}, \ldots, p_{n+q}\right\}$ such that $p_{i}=1$. Since the proof process here is also analogous to the corresponding part in the proof of Theorem 1.1, then we omit it here, Theorem 1.3 is proved.

\subsection{Proof of Theorem 1.5}

Proof Let $\sigma_{i}=1+s\left(1-\frac{1}{p_{i}}\right) \geq 1$ and $v_{i}(r, x, t)=\left(r^{\frac{n+2}{s}-\bar{\gamma}} t^{\frac{n+2}{s}-\gamma}|u(r, x, t)|\right)^{\sigma_{i}}$ for $0 \leq i \leq n+1$. Due to $\frac{1}{s}=\frac{1}{n+2}\left(\sum_{i=0}^{n+1} \frac{1}{p_{i}}-1\right)$, we have $\sum_{i=0}^{n+1} \sigma_{i}=(n+1) s$.

Since $u(r, x, t) \in C_{0}^{\infty}\left(\mathbb{R}_{+} \times \mathbb{R}^{n} \times \mathbb{R}_{+}\right)$, then we obtain, for $i=0$ and $r>0$,

$$
2 v_{0}(r, x, t)=\int_{0}^{r}\left(\tau \partial_{\tau}\right) v_{0}(\tau, x, t) \frac{\mathrm{d} \tau}{\tau}-\int_{r}^{+\infty}\left(\tau \partial_{\tau}\right) v_{0}(\tau, x, t) \frac{\mathrm{d} \tau}{\tau}
$$

Thus

$$
\begin{aligned}
\left|2 v_{0}(r, x, t)\right| & \leq \int_{0}^{r}\left|\left(\tau \partial_{\tau}\right) v_{0}(\tau, x, y)\right| \frac{\mathrm{d} \tau}{\tau}+\int_{r}^{+\infty}\left|\left(\tau \partial_{\tau}\right) v_{0}(\tau, x, t)\right| \frac{\mathrm{d} \tau}{\tau} \\
& =\int_{0}^{+\infty}\left|\left(r \partial_{r}\right) v_{0}(r, x, t)\right| \frac{\mathrm{d} r}{r} .
\end{aligned}
$$

For $1 \leq i \leq n$, we obtain, for $r, t \in \mathbb{R}_{+}$,

$$
\begin{aligned}
\left|2 v_{i}(r, x, t)\right| \leq & \int_{-\infty}^{x_{i}}\left|\partial_{\hat{x}_{i}} v_{i}\left(r, x_{1}, x_{2}, \ldots, x_{i-1}, \hat{x}_{i}, x_{i+1}, \ldots, x_{n}, t\right)\right| \mathrm{d} \hat{x}_{i} \\
& +\int_{x_{i}}^{+\infty}\left|\partial_{\hat{x}_{i}} v_{i}\left(r, x_{1}, x_{2}, \ldots, x_{i-1}, \hat{x}_{i}, x_{i+1}, \ldots, x_{n}, t\right)\right| \mathrm{d} \hat{x}_{i} \\
= & \int_{-\infty}^{+\infty}\left|\partial_{x_{i}} v_{i}(r, x, t)\right| \mathrm{d} x_{i} .
\end{aligned}
$$


Similarly, for $r, t>0$,

$$
\begin{aligned}
\left|2 v_{n+1}(r, x, t)\right| & \leq \int_{0}^{t}\left|\left(r \mu \partial_{\mu}\right) v_{n+1}(r, x, \mu)\right| \frac{\mathrm{d} \mu}{r \mu}+\int_{t}^{+\infty}\left|\left(r \mu \partial_{\mu}\right) v_{n+1}(r, x, \mu)\right| \frac{\mathrm{d} \mu}{r \mu} \\
& =\int_{0}^{+\infty}\left|\left(r \mu \partial_{\mu}\right) v_{n+1}(r, x, \mu)\right| \frac{\mathrm{d} \mu}{r \mu} \\
& =: \int_{0}^{+\infty}\left|\left(r t \partial_{t}\right) v_{n+1}(r, x, t)\right| \frac{\mathrm{d} t}{r t},
\end{aligned}
$$

where $x=\left(x_{1}, x_{2}, \ldots, x_{n}\right) \in \mathbb{R}^{n}$. By multiplying the $n+2$ inequalities above, we have

$$
\begin{aligned}
\prod_{i=0}^{n+1}\left|2 v_{i}(r, x, t)\right|= & 2^{n+2}\left(r^{\frac{n+2}{s}-\bar{\gamma}} t^{\frac{n+2}{s}-\gamma}|u(r, x, t)|\right)^{s(n+1)} \\
\leq & \int_{0}^{+\infty}\left|\left(r \partial_{r}\right) v_{0}(r, x, t)\right| \frac{\mathrm{d} r}{r} \prod_{i=1}^{n} \int_{-\infty}^{+\infty}\left|\partial_{x_{i}} v_{i}(r, x, t)\right| \mathrm{d} x_{i} \\
& \times \int_{0}^{+\infty}\left|\left(r t \partial_{t}\right) v_{n+1}(r, x, t)\right| \frac{\mathrm{d} t}{r t} .
\end{aligned}
$$

That means

$$
\begin{aligned}
2^{\frac{n+2}{n+1}} & \left(r^{\frac{n+2}{s}-\bar{\gamma}} t^{\frac{n+2}{s}-\gamma}|u(r, x, t)|\right)^{s} \\
\leq & \left(\int_{0}^{+\infty}\left|\left(r \partial_{r}\right) v_{0}(r, x, t)\right| \frac{\mathrm{d} r}{r}\right)^{\frac{1}{n+1}} \prod_{i=1}^{n}\left(\int_{-\infty}^{+\infty}\left|\partial_{x_{i}} v_{i}(r, x, t)\right| \mathrm{d} x_{i}\right)^{\frac{1}{n+1}} \\
& \times\left(\int_{0}^{+\infty}\left|\left(r t \partial_{t}\right) v_{n+1}(r, x, t)\right| \frac{\mathrm{d} t}{r t}\right)^{\frac{1}{n+1}} .
\end{aligned}
$$

Now integrating over the interval $(0,+\infty)$ with respect to $\frac{\mathrm{d} r}{r}$ and $\frac{\mathrm{d} t}{r t}$ and using Hölder's inequality respectively, we obtain

$$
\begin{aligned}
2^{\frac{n+2}{n+1}} & \int_{0}^{+\infty} \int_{0}^{+\infty}\left(r^{\frac{n+2}{s}-\bar{\gamma}} t^{\frac{n+2}{s}-\gamma}|u(r, x, t)|\right)^{s} \frac{\mathrm{d} r}{r} \frac{\mathrm{d} t}{r t} \\
\leq & \left(\int_{0}^{+\infty} \int_{0}^{+\infty}\left|\left(r \partial_{r}\right) \nu_{0}(r, x, t)\right| \frac{\mathrm{d} r}{r} \frac{\mathrm{d} t}{r t}\right)^{\frac{1}{n+1}}\left(\int_{0}^{+\infty} \int_{0}^{+\infty}\left|\left(r t \partial_{t}\right) v_{n+1}(r, x, t)\right| \frac{\mathrm{d} t}{r t} \frac{\mathrm{d} r}{r}\right)^{\frac{1}{n+1}} \\
& \times \prod_{i=1}^{n}\left(\int_{0}^{+\infty} \int_{0}^{+\infty} \int_{-\infty}^{+\infty}\left|\partial_{x_{i}} v_{i}(r, x, t)\right| \mathrm{d} x_{i} \frac{\mathrm{d} r}{r} \frac{\mathrm{d} t}{r t}\right)^{\frac{1}{n+1}} .
\end{aligned}
$$

Then from integrating over the interval $(-\infty,+\infty)$ with $x_{1}, x_{2}, \ldots, x_{n}$ respectively and using Hölder's inequality again, we derive that

$$
\begin{gathered}
2^{\frac{n+2}{n+1}} \int_{\mathbb{R}_{+}} \int_{\mathbb{R}^{n}} \int_{\mathbb{R}_{+}}\left(r^{\frac{n+2}{s}-\bar{\gamma}} t^{\frac{n+2}{s}-\gamma}|u(r, x, t)|\right)^{s} \frac{\mathrm{d} r}{r} \mathrm{~d} x \frac{\mathrm{d} t}{r t} \\
\leq\left(\int_{\mathbb{R}_{+}} \int_{\mathbb{R}^{n}} \int_{\mathbb{R}_{+}}\left|\left(r \partial_{r}\right) \nu_{0}(r, x, t)\right| \frac{\mathrm{d} r}{r} \mathrm{~d} x \frac{\mathrm{d} t}{r t}\right)^{\frac{1}{n+1}}
\end{gathered}
$$




$$
\begin{aligned}
& \times\left(\int_{\mathbb{R}_{+}} \int_{\mathbb{R}^{n}} \int_{\mathbb{R}_{+}}\left|\left(r t \partial_{t}\right) v_{n+1}(r, x, t)\right| \frac{\mathrm{d} r}{r} \mathrm{~d} x \frac{\mathrm{d} t}{r t}\right)^{\frac{1}{n+1}} \\
& \times \prod_{i=1}^{n}\left(\int_{\mathbb{R}_{+}} \int_{\mathbb{R}^{n}} \int_{\mathbb{R}_{+}}\left|\partial_{x_{i}} v_{i}(r, x, t)\right| \frac{\mathrm{d} r}{r} \mathrm{~d} x \frac{\mathrm{d} t}{r t}\right)^{\frac{1}{n+1}}
\end{aligned}
$$

Set $\mathrm{d} \eta:=\frac{\mathrm{d} r}{r} \mathrm{~d} x \frac{\mathrm{d} t}{r t}$, and $N=n+2$. Similar to the estimation in Theorem 1.3, we acquire that $\left|\partial_{x_{i}}\right| u(r, x, t)|| \leq\left|\partial_{x_{i}} u(r, x, t)\right|$ for $1 \leq i \leq n,\left|\left(r \partial_{r}\right)\right| u(r, x, t)|| \leq\left|\left(r \partial_{r}\right) u(r, x, t)\right|$ and $\left|\left(r t \partial_{t}\right)\right| u(r, x, t)|| \leq\left|\left(r t \partial_{t}\right) u(r, x, t)\right|$. As a result,

$$
\begin{aligned}
\left|\partial_{x_{i}} \nu_{i}(r, x, t)\right| & =\left|\partial_{x_{i}}\left(r^{\frac{n+2}{s}-\bar{\gamma}} t^{\frac{n+2}{s}-\gamma}|u(r, x, t)|\right)^{\sigma_{i}}\right| \\
& \leq \sigma_{i}\left(r^{\frac{n+2}{s}-\bar{\gamma}} t^{\frac{n+2}{s}-\gamma}|u(r, x, t)|\right)^{\sigma_{i}-1} r^{\frac{n+2}{s}-\bar{\gamma}} t^{\frac{n+2}{s}-\gamma}\left|\partial_{x_{i}} u(r, x, t)\right|
\end{aligned}
$$

for $1 \leq i \leq n$,

$$
\begin{aligned}
& \left|\left(r \partial_{r}\right) v_{0}(r, x, t)\right| \\
& \quad \leq \sigma_{0}\left(r^{\frac{n+2}{s}-\bar{\gamma}} t^{\frac{n+2}{s}-\gamma}|u(r, x, t)|\right)^{\sigma_{0}-1}\left[\left|\frac{n+2}{s}-\bar{\gamma}\right| r^{\frac{n+2}{s}-\bar{\gamma}} t^{\frac{n+2}{s}-\gamma}|u(r, x, t)|\right. \\
& \left.\quad+r^{\frac{n+2}{s}-\bar{\gamma}} t^{\frac{n+2}{s}-\gamma}\left|\left(r \partial_{r}\right) u(r, x, t)\right|\right]
\end{aligned}
$$

and

$$
\begin{aligned}
& \left|\left(r t \partial_{t}\right) v_{n+1}(r, x, t)\right| \\
& \leq \sigma_{n+1}\left(r^{\frac{n+2}{s}-\bar{\gamma}} t^{\frac{n+2}{s}-\gamma}|u(r, x, t)|\right)^{\sigma_{n+1}-1}\left[\left|\frac{n+2}{s}-\gamma\right| r^{\frac{n+2}{s}-\bar{\gamma}+1} t^{\frac{n+2}{s}-\gamma}\right. \\
& \left.\quad \times|u(r, x, t)|+r^{\frac{n+2}{s}-\bar{\gamma}} t^{\frac{n+2}{s}-\gamma}\left|\left(r t \partial_{t}\right) u(r, x, t)\right|\right]
\end{aligned}
$$

Substituting (4.6), (4.7), and (4.8) into (4.5), it is easy to see

$$
\begin{aligned}
2^{\frac{n+2}{n+1}} & \int_{\mathbb{R}_{+}^{N}}\left(r^{\frac{n+2}{s}-\bar{\gamma}} t^{\frac{n+2}{s}-\gamma}|u(r, x, t)|\right)^{s} \mathrm{~d} \eta \\
\leq & {\left[\sigma_{0}\left|\frac{n+2}{s}-\bar{\gamma}\right| \int_{\mathbb{R}_{+}^{N}}\left(r^{\frac{n+2}{s}-\bar{\gamma}} t^{\frac{n+2}{s}-\gamma}|u(r, x, t)|\right)^{\sigma_{0}-1}\left(r^{\frac{n+2}{s}-\bar{\gamma}} t^{\frac{n+2}{s}-\gamma}|u(r, x, t)|\right) \mathrm{d} \eta\right.} \\
& \left.+\sigma_{0} \int_{\mathbb{R}_{+}^{N}}\left(r^{\frac{n+2}{s}-\bar{\gamma}} t^{\frac{n+2}{s}-\gamma}|u(r, x, t)|\right)^{\sigma_{0}-1}\left(r^{\frac{n+2}{s}-\bar{\gamma}} t^{\frac{n+2}{s}-\gamma}\left|\left(r \partial_{r}\right) u(r, x, t)\right|\right) \mathrm{d} \eta\right]^{\frac{1}{n+1}} \\
& \times \prod_{i=1}^{n}\left(\int_{\mathbb{R}_{+}^{N}} \sigma_{i}\left(r^{\frac{n+2}{s}-\bar{\gamma}} t^{\frac{n+2}{s}-\gamma}|u(r, x, t)|\right)^{\sigma_{i}-1}\left(r^{\frac{n+2}{s}-\bar{\gamma}} t^{\frac{n+2}{s}-\gamma}\left|\partial_{x_{i}} u(r, x, t)\right|\right) \mathrm{d} \eta\right)^{\frac{1}{n+1}} \\
& \times\left[\sigma_{n+1}\left|\frac{n+2}{s}-\gamma\right| \int_{\mathbb{R}_{+}^{N}}\left(r^{\frac{n+2}{s}-\bar{\gamma}} t^{\frac{n+2}{s}-\gamma}|u(r, x, t)|\right)^{\sigma_{n+1}-1}\right. \\
& \times\left(r^{\frac{n+2}{s}-\bar{\gamma}+1} t^{\frac{n+2}{s}-\gamma}|u(r, x, t)|\right) \mathrm{d} \eta+\sigma_{n+1} \int_{\mathbb{R}_{+}^{N}}\left(r^{\frac{n+2}{s}-\bar{\gamma}} t^{\frac{n+2}{s}-\gamma}|u(r, x, t)|\right)^{\sigma_{n+1}-1}
\end{aligned}
$$




$$
\begin{aligned}
& \left.\times\left(r^{\frac{n+2}{s}-\bar{\gamma}} t^{\frac{n+2}{s}-\gamma}\left|\left(r t \partial_{t}\right) u(r, x, t)\right|\right) \mathrm{d} \eta\right]^{\frac{1}{n+1}} \\
= & \left(I_{1}+I_{2}\right)^{\frac{1}{n+1}} \cdot I_{3}\left(I_{4}+I_{5}\right)^{\frac{1}{n+1}} .
\end{aligned}
$$

Considering that the remaining proofs will be the same as those in both Theorem 1.1 and Theorem 1.3, then Theorem 1.5 is proved.

Acknowledgements

Not applicable.

Funding

This work is supported by the National Natural Science Foundation of China (Grants Nos. 11631011 and 11626251 ).

Availability of data and materials

Not applicable.

\section{Competing interests}

The authors declare that they have no competing interests.

\section{Authors' contributions}

$\mathrm{HC}$ first raised the core problem of the current paper. Under his supervision and suggestion, YL and JW finished this manuscript together. Then $\mathrm{HC}$ read carefully this manuscript for several times and gave some valuable revisions on it. All authors read and approved the final manuscript.

\section{Publisher's Note}

Springer Nature remains neutral with regard to jurisdictional claims in published maps and institutional affiliations.

Received: 20 June 2019 Accepted: 1 October 2019 Published online: 10 October 2019

\section{References}

1. Troisi, M.: Teoremi di inclusione per spazi di Sobolev non isotropi. Ric. Mat. 18(1), 3-24 (1969)

2. El Hamidi, A., Rakotoson, J.: Extremal functions for the anisotropic Sobolev inequalities. Ann. Inst. Henri Poincaré, Anal. Non Linéaire 24, 741-756 (2007)

3. Cirstea, F.C., Vétois, J.: Fundamental solutions for anisotropic elliptic equations: existence and a priori estimates. Commun. Partial Differ. Equ. 40(4), 727-765 (2015)

4. Guo, Z., Caggio, M., Skalák, Z.: Regularity criteria for the Navier-Stokes equations based on one component of velocity. Nonlinear Anal., Real World Appl. 35, 379-396 (2017)

5. Adams, R.A.: Anisotropic Sobolev inequalities. Čas. Pěst. Mat. 113, 267-279 (1988)

6. Kružkov, S.: Boundary value problems for degenerate second order elliptic equations. Sb. Math. 6(3), 275-307 (1968)

7. Chen, H., Liu, X., Wei, Y.: Cone Sobolev inequality and Dirichlet problem for nonlinear elliptic equations on a manifold with conical singularities. Calc. Var. Partial Differ. Equ. 43(3-4), 463-484 (2012)

8. Chen, H., Liu, X., Wei, Y.: Dirichlet problem for semilinear edge-degenerate elliptic equations with singular potential term. J. Differ. Equ. 252, 4289-4313 (2012)

9. Chen, H., Liu, X., Wei, Y.: Multiple solutions for semi-linear corner degenerate elliptic equations. J. Funct. Anal. 266(6), 3815-3839 (2014)

\section{Submit your manuscript to a SpringerOpen ${ }^{\odot}$ journal and benefit from:}

$\checkmark$ Convenient online submission

- Rigorous peer review

Open access: articles freely available online

- High visibility within the field

- Retaining the copyright to your article 